\title{
Etude du périphyton d'un secteur pollué d'une rivière varoise, l'Argens. Evolution spatiale du peuplement algal en période d'intense pollution
}

\author{
N. Galvin-Chabrière ${ }^{1}$ \\ A. Cazaubon ${ }^{1}$
}

L'étude dont nous présentons les résultats, a eu pour but de déterminer les perturbations subies par un cours d'eau sous l'effet d'une pollution organique provoquée par des rejets urbains et industriels.

L'évolution de la charge polluante ainsi que son impact sur les principaux constituants abiotiques et biotiques de l'écosystème aquatique ont été analysés au travers de mesures mensuelles des composantes physico-chimiques et de prélèvements du périphyton.

Les évolutions de la production algale. de la composition et de la structure du périphyton ont été suivies le long d'un secteur de $10 \mathrm{~km}$ à l'aide de substrats artificiels renouvelés tous les 12 jours.

Dans la zone étudiée, on observe le passage progressif d'un peuplement algal de faible diversité spécifique, composé essentieliement d'Euglénophycées, de Chlorophycées et de Cyanophycées, en milieu très pollué, à un peuplement diversifié dominé par les Diatomées en aval de ce secteur.

Ce travail montre que le peuplement algal, intégrateur des conditions de milieu, traduit par sa composition et sa struc. ture les variations de la qualité de l'eau et reflète ainsi l'évolution spatiale des conditions hydrochimiques.

A study of pertphyton in a polluted section of a Varoise river, the Argens. Spatial evolution of the algal population during a period of intense pollution.

The objectives of this study were to follow changes in a water body that was subjected to organic pollution from urban and industrial effluents.

The evolution of the pollution load as well as its impact on the chief abiotic and biotic parts of the aquatic ecosystem were studied by taking monthly readings of physical-chemical variables and samples of periphyton.

Changes in algal production, and the composition and structure of the periphyton were followed in a $10 \mathrm{~km}$ stretch by using artificial substrata that were renewed every 12 days.

In the study zone, there was a progressive change from an algal population of low diversity (chiefly Euglenophyceae, Chlorophyceae and Cyanophyceae) in the polluted environment, to a diverse population dominated by diatoms in the section downstream.

This investigation shows that algal populations integrate the environmental conditions and show through their composition and structure the variations in water quality, thus reflecting the spatial changes in hydrochemical conditions.

\section{1. - Introduction}

Les recherches réalisées sur l'Argens depuis 1976 ont porté sur l'hydrologie et l'hydrochimie, sur l'étude de l'organisation des communautés d'invertébrés benthiques (Martinez-Ansemil 1978) et leur

\footnotetext{
1. Laboratoire de Biołogie Animale et Ecologie, Faculté des sciences et Techniques de St Jérôme. Universite d'Aix-Marséilie Ill, rue Henri Poincare, 13397 Marseille Cedex 13.
}

zonation longitudinale (Giudicelli, Dia \& Legier 1980).

On a reconnu dans le cours superieur de la rivière :

- l'existence d'une zone atypique constituée par les marais de l'Argens.

- la présence de nombreux foyers de pollution organique,

- l'impact de ces pollutions sur les communautés animales benthiques. 
A la suite de ces travaux nous avons entrepris de déterminer les perturbations que subissent, dans le haut Argens, les communautés périphytiques sous l'effet de ces pollutions organiques.

A cet effet, nous avons pris en compte le peuple. ment algal dans le secteur ou il reçoit les charges organiques les plus importantes; dans ce même secteur se trouvent réalisées, en outre, des conditions hydrologiques propices au développement d'un riche périphyton du fait de l'écoulement ralenti et de la formation de zones palustres.

\section{2. - Le milieu}

L'Argens, rivière du département du Var, prend sa source sur la commune de Seillons à l'Est du massif de la Sainte-Victoire et se jette, après un parcours de $114 \mathrm{Km}$, dans le Golfe de Fréjus.

Le secteur prospecté, long de $10 \mathrm{~km}$, se localise dans le cours supérieur (fig. 1); il comprend, d'une part, le système des sources de l'Argens (Meyronne,
Sceaux, Argens) et leurs émissaires s'écoulant sur des terrains calcaires et, d'autre part, une zone d'eau calme et d'alluvionnement formée par la confluence de ces émissaires.

Les rejets domestiques de la ville de SaintMaximin (4 000 habitants) et les rejets de coopéra. tives vinicoles constituent à l'étiage le principal foyer de pollution du haut Argens. Les eaux usées sont déversées en totalité dans le ruisseau des Fontaines qui, après un parcours de $4 \mathrm{~km}$, se jette dans la Meyronne. Celle-ci, après avoir recueilli les eaux de la source de Sceaux, débouche dans l'Argens. Au niveau de la confluence de ces deux émissaires, la vallée s'élargit et la rivière forme sur une distance de $2.3 \mathrm{Km}$ une zone à écoulement ralenti qui constitue les "marais de l'Argens" ou se développe une végétation macrophytique dense, dominée par Typha latifolia. Le lit se resserre en aval.

L'étude a porté sur la période de l'année où les rejets sont les plus abondants, d'août à décembre, lorsque le milieu aquatique reçoit conjointement les rejets urbains et les rejets des coopératives.

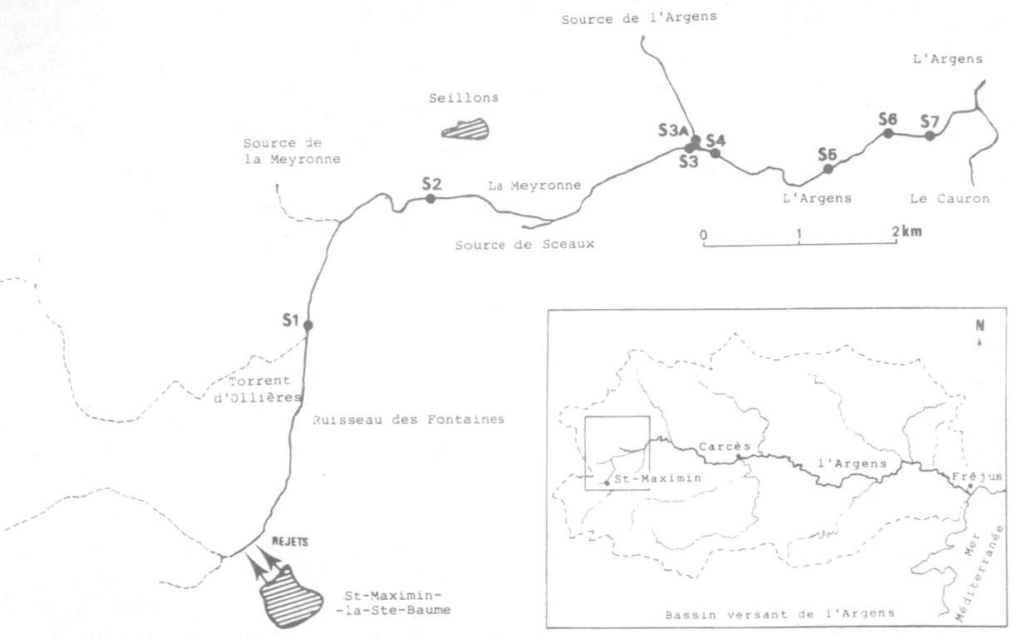

FIG 1. Localisation des stations d'étude. 
Huit stations ont été prospectées :

- les stations S1, S2, S3 sont situées sur des affluents du haut Argens, respectivement à $2,3 \mathrm{Km}$. $4,6 \mathrm{Km}, 7,6 \mathrm{Km}$ des rejets. Dans cette zone, l'activité agricole est intense et de nombreux vignobles longent les rives.

- La station S3A est localisée sur l'Argens, à $2 \mathrm{Km}$ de sa source, en amont de la confluence de cette rivière avec la Meyronne.

- Les stations S4, S5, S6 se succedent dans le marais.

- La station S7 est située en aval du marais; les macrophytes y sont absents; les rives sont occupees par une ripisylve dense.

Lors de l 'étiage, les débits moyens stationnels oscillent entre $110 \mathrm{l} / \mathrm{s}$ en $S 1$ et $345 \mathrm{l} / \mathrm{s}$ dans le marais. La vitesse est de l'ordre de $10 \mathrm{~cm} / \mathrm{s}$, excepté en $\mathrm{S7}$ où elle atteint $60 \mathrm{~cm} / \mathrm{s}$. Dans l'ensemble des stations la hau teur d'eau n'excède pas $1,50 \mathrm{~m}$.

Des analyses chimiques ont été réalisées chaque mois dans chaque station.

L'impact des rejets urbains organiques se traduit dans les deux premières stations (S1, S2) par un déficit en oxygène dissous (les taux de saturation sont inférieurs à $16 \%$ ), et par un enrichissement des eaux en $\mathrm{PO}_{4}-\mathrm{P}(1$ à $12 \mathrm{mg} / \mathrm{l})$, en $\mathrm{NH}_{4}-\mathrm{N}(6,9$ à $43 \mathrm{mg} / \mathrm{l})$, en matières organiques particulaires (MOP $=9$ à 53 $\mathrm{mg} / \mathrm{l}$ ). L'oxydabilité (matières organiques dissoutes $=$ MOD) est élevée et oscille entre 5,5 et $30,5 \mathrm{mg} \mathrm{d} \mathrm{O}_{2} / \mathrm{l}$.

En aval, les teneurs en oxygène dissous augmentent alors que les concentrations en $\mathrm{PO}_{4}-\mathrm{P}$. $\mathrm{NH}_{\mathbf{4}}-\mathrm{N}, \mathrm{MOP}$ et l'oxydabilité diminuent. Ainsi, dans la portion de l'Argens comprise entre S3 et S7, la moyenne des taux de saturation passe de $53 \%$ à $60 \%$ : les valeurs moyennes de $\mathrm{PO}_{4}-\mathrm{P}$ varient entre 0,48 et $0,21 \mathrm{mg} /$, celles de $\mathrm{NH}_{4}-\mathrm{N}$ entre 1,42 et $0,27 \mathrm{mg} / \mathrm{l}$, celles de l'oxydabilité et de MOP variant respectivement de 1,81 à $1,22 \mathrm{mg} \mathrm{d} \mathrm{O}_{2} / \mathrm{l}$ et de 2,75 à $2,30 \mathrm{mg} / \mathrm{l}$.

Dans ce secteur, les sels d'azote se manifestent aussi sous forme de nitrites $(0,070$ à $0,044 \mathrm{mg} / 1$ de $\left.\mathrm{NO}_{2}-\mathrm{N}\right)$ et de nitrates $(0,132$ à $0,090 \mathrm{mg} / 1$ de $\mathrm{NO}_{3}-\mathrm{N}$ ).

Au niveau de la station $\mathbf{S} 3 \mathrm{~A}$, les pourcentages de saturation sont élevés ( 74 à $85 \%$ ), les éléments phosphatés et azotés sont absents et les valeurs moyennes de l'oxydabilité $\left(0,92 \mathrm{mg} \mathrm{d} \mathrm{O}_{2} / \mathrm{l}\right)$ et des MOP $(2,85$ $\mathrm{mg} / \mathrm{l})$ sont réduites.
Dans l'ensemble du secteur étudié, les teneurs en $\mathrm{Ca}^{++}, \mathrm{Mg}^{++}$sont stables, respectivement de l'ordre de 105 et $18 \mathrm{mg} / \mathrm{l}$; les teneurs moyennes en sulfates passent de $31 \mathrm{mg} / \mathrm{l}$ en $\mathrm{S} 1$ à $17 \mathrm{mg} / \mathrm{l}$ en $\mathrm{S} 7$, les valeurs de l'alcalinité varient entre 291 et $465 \mathrm{mg} \mathrm{\#}$ $\mathrm{CaCO}_{3} / \mathrm{I}$ dans les deux premières stations et avolsinent $310 \mathrm{mg} / \mathrm{l}$ dans les suivantes.

Dans l'ensemble des stations le $\mathrm{pH}$ varie entre 7,2 et 7,9 .

\section{3. - Méthodes d'étude du peuplement algal}

Dans le secteur prospecté le peuplement algal est essentiellement représenté par le périphyton.

\section{1 - Méthodes de terrain}

Nous avons employé des substrats artificiels du type de ceux utilisés par Capblancq \& Cassan (1979).

Ces substrats sont constitués par une feuille de polyéthylène transparent de $0,1 \mathrm{~mm}$ d'épaisseur, enroulée sur un cylindre en P.V.C. de $7,5 \mathrm{~cm}$ de diamètre et de $22 \mathrm{~cm}$ de hauteur. Ils sont lestés et maintenus en position verticale dans l'eau par un flotteur. La récolte concerne ainsi les vingt-cinq premiers centimètres de la masse d'eau.

Au cours de la période d'intense pollution (d'août à décembre 1980) un substrat a été mis en place dans chaque station, prélevé au bout de douze jours et renouvelé avec la même fréquence.

La colonisation des substrats étant rapide, cette périodicité (12 jours) peut être considérée comme suffisante pour être significative.

Chaque substrat reçoit directement le rayonnement solaire, sauf à la station 7 où le couvert végétal dense limite la pénétration de la lumière. Dans cette dernière station, nous avons choisi un secteur calme où la vitesse du courant n'excède pas $10 \mathrm{~cm} / \mathrm{s}$; ainsi, tous les substrats quelle que soit la station, sont soumis au mème courant.

Lors du prélèvement, la feuille de polyéthylène recouverte par le périphyton est découpée en trois bandes rectangulaires, mesurant environ $150 \mathrm{~cm}^{2}$; ces trois échantillons sont exploités ainsi :

- le premier, destiné aux dosages des pigments chlorophylliens, est conservé dans de l'eau additionnée de trois gouttes de $\mathrm{MgCO}_{3}(10 \%)$, maintenu à l'obscurité et au frais dans une glacière. 
- le second, conservé dans du formol, est destiné aux mesures gravimétriques dont les résultats ne sont pas exprimes dans ce travail

- le troisième, fixé par du formol et du lugol, est destiné à l'examen microscopique du périphyton.

\section{2 - Méthodes de laboratoire}

\section{- Dosage des pigments}

Le périphyton, recueilli par brossage et lavage du premier échantillon. est mis en suspension dans l'eau puis homogénéisé. Deux échantillons de $25 \mathrm{~cm}^{3}$ et $50 \mathrm{~cm}^{3}$ sont alors prélevés pour être filtrés sur filtre Whatman GFC $(1,2 \mu \mathrm{m})$.

L'extraction des pigments est réalisée en immergeant, durant $\mathbf{2 4}$ heures, ces filtres dans l'acétone $(90 \%)$, à l'obscurité et au frais.

L'ensemble de ces opérations est effectué le soir même des prélèvements ou, au plus tard, le lendemain matin.

Les densités optiques de ces extraits sont mesurées grâce à un spectrophotomètre pour les longueurs d'onde caractéristiques de $750 \mathrm{~nm}$ et $665 \mathrm{~nm}$ (avant et après acidification), $645 \mathrm{~nm}$ et $630 \mathrm{~nm}$, ce qui permet de calculer les teneurs en chlorophylle "a" active d'après l'équation de Lorenzen (1967), et celles des chlorophylles ab" et "c" à partir des équations de SCOR-UNESCO (1964) (in Millerioux 1974-1975).

\section{- Determination et COMPTAGe des algues}

En différents points de la feuille de polyéthylène, plusieurs carrés d'un centimètre carré (surface significative pour le comptage) sont découpés. Chaque carré est débarrassé de son bioderme par un grattage complet au moyen d'un scalpel. Cet échantillon d'algues est aussitôt placé sur une lame histologique dans une goutte d'eau distillée, puis recouvert d'une lamelle. Les cellules algales sont dénombrées et déterminées au microscope (grossissemenı 100 par immersion). L'effectif moyen de ces algues est ensuite établi pour un centimètre carré de substrat.

Ces données d'échantillonnage nous ont permis de déterminer la composition et la structure des com. munautés algales et d'analyser leurs variations spatiales.

\section{4. - Composition et structure des communautés algales}

\section{1 - Plgments chlorophylliens et densités algales}

Les teneurs en chlorophylle "a* oscillent entre 3 ${ }_{\mu} \mathrm{g} / \mathrm{dm}^{2}$ et $1530{ }_{\mu} \mathrm{g} / \mathrm{dm}^{2}$ (fig. 2) et présentent en S2 les valeurs maximales.

Les vitesses d'accumulation de la chlorophylle "a", comprises entre $0,027 \mathrm{mg} / \mathrm{m}^{2} / \mathrm{j}$ et $12,75 \mathrm{mg} / \mathrm{m}^{2} / \mathrm{j}$, sont généralement supérieures à celles mesurées par Capblancq et Cassan (1979) en milieu pollué.

La proportion de chlorophylle "b" est élevée en S1 et S2, zone fortement polluée, et s'abaisse en aval alors que croît la proportion de chlorophylle "c" (fig. 3).

La densité algale stationnelie représente le nombre de cellules dénombrées au cours de chaque prélèvement dans une station donnée. Sur la figure 2, où sont illustrées les variations spatio-temporelles des taux de chlorophylle «a» et des densités, on constate que les deux paramètres varient globalement dans le méme sens et que les concentrations de chlorophylle "aw donnent une bonne image de la production algale.

Les densités et les teneurs en chlorophylle "an sont généralement élevées dans les stations polluées S1, S2 et dans le marais \$5, S6.

Il faut analyser de près ces résultats car, si les taux de chlorophylle "a" donnent une bonne représentation de la biomasse algale, par contre des valeurs identiques de chlorophylle «an ne correspondent pas toujours à des peuplements de même composition floristique (fig. 2).

Ainsi dans les stations $\mathrm{S} 1$ et $\mathrm{S} 2$ qui présentent des teneurs élevées en $\mathrm{PO}_{4}-\mathrm{P}, \mathrm{NH}_{4}-\mathrm{N}$, MOD et des teneurs faibles en oxygène dissous, les fortes valeurs de la chlorophylle "a" correspondent au developpement des Cyanophycées, des Chlorophycées et/ou des Euglénophycées. Les taux élevés de chlorophylle "b» dans ces stations témoignent donc de la présence d'un ou des deux derniers groupes dans le peuplement et traduisent l'importance de ces groupes par rapport à celle des Chrysophytes.

Dans les stations situées dans le marais, caractérisées par des teneurs en $\mathrm{PO}_{4}-\mathrm{P}, \mathrm{NH}_{4}-\mathrm{N}$, MOD plus faibles qu'en $\mathbf{S 1}, \mathbf{S} 2$ mais par des concentrations en $\mathrm{NO}_{2}-\mathrm{N}, \mathrm{NO}_{3}-\mathrm{N}, \mathrm{O}_{2}$ supérieures, les taux élevés de chlorophylle «a” et la forte proportion de chlo- 

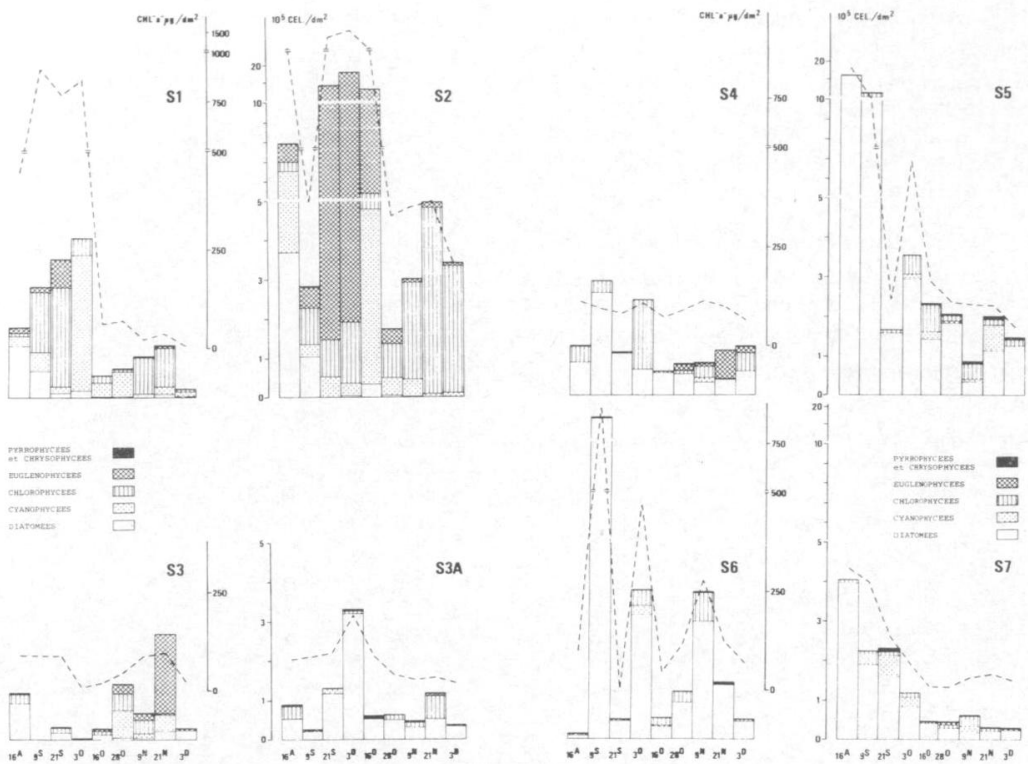

FIG 2. Evolution stationnelle du 16 août au 3 décembre : - des teneurs en chlorophylle "an active (exprimées en $\mu \mathrm{g} / \mathrm{dm}^{2}$ traits en tireté)

- du nombre total de cellules végétales réparties dans les classes d'algues (histogramme).

Les interruptions de graphisme correspondent à un changement d'échelle.

FIG 3. Pourcentages stationnels de $\frac{\mathrm{Chl} \mathrm{c} \text { : }}{\mathrm{Chl} \mathrm{c+Chl} \mathrm{b}}$

- moyennes et amplitudes de variations.
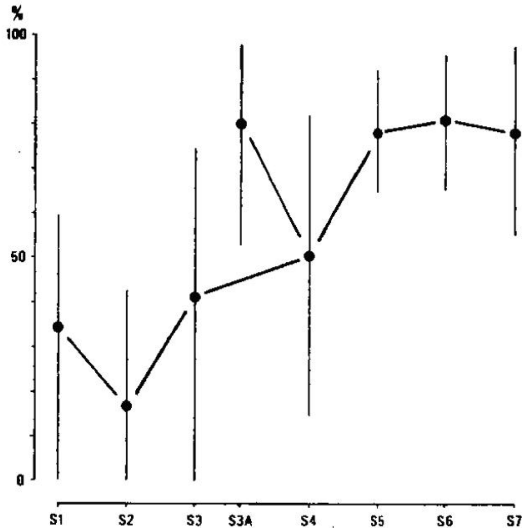
rophylle «c" reflètent essentiellement le peuplement diatomique.

Dans les autres stations ou les concentrations en chlorophylle "a" sont réduites, les populations algales, peu abondantes, sont représentées principalement par les Diatomées en S3A et S7, et par l'ensemble de groupes en $\mathrm{S} 3$ et $\mathrm{S} 4$.

\section{2 - Inventaire et composition du peuplement}

Les 135 espèces dénombrées dans l'ensemble du secteur étudié sont récapitulées ci-après ; le groupe des Diatomées est le mieux représenté ( 91 espèces).

\section{EUCHLOROPHYCEES}

Chlamydomonas $s p$.

Carleria sp.

Pandorina sp.

Eudorina sp.

Volvox $s p$.

Tetraspora sp.

Chlorella sp.

Eremosphaera sp.

Ankistrodesmus sp.

Coelastrum microporum NÄG

Micractinium sp.

Dictyosphaerium sp.

Scenedesmus sp.

Hydrodictyon $s p$.

\section{ULOTHRICOPHYCEES}

Ulothrix sp.

Microspora sp.

Stigeoclonium tenue KUTZ

Protoderma sp.

Pseudulvella $s p$.

Oedogonium sp.

\section{ZYGOPHYCEES}

Mougeotia sp.

Mesotaenium sp.

Closterium $s p$.

Penium sp.

Cosmarium sp.

\section{CHRYSOPHYCEES}

Chrysococcus sp.

Dinobryon $s p$.

Synura sp.

Rhipidodendron $s p$.

\section{XANTHOPHYCEES}

Tribonema sp.

\section{DIATOMOPHYCEES}

Melosira varians C.A. AGARDH

Melosira granulata (EHR.) RALFS

Cyclotella sp.

Cyclotella meneghiniana KUTZ

Cyclotella austriaca (PERAG.) HUSTEDT

Stephanodiscus hantzschii GRUN

Thalassiosira weissflogii GRUN

Coscinodiscus $s p$.

Diatoma vulgare BORY

Dialoma elongatum (LYNGBYE) AGARDH

Diatoma hiemale (LYNGBYE) HEIBERG

Centronella reicheltii VOIGT

Asterionella formosa HASSAL

Fragilaria construens (EHR.) GRUN

Fragilaria virescens RALFS

Synedra ulna (NITZSCHE) EHR

Synedra pulchella KUTZ

Synedra tabulata AGARDH

Synedra rumpens KUTZ

Eunotia robusta RALFS

Eunotia faba (EHR.) GRUN

Eunotia parallela EHR

Cocconeis placentula EHR

Cocconeis placentula var euglypta (EHR.) CLEVE

Achnanthes microcephala KUTZ

Achnanthes inflata KUTZ

Rhoicosphenia curvata (KUTZ) GRUN

Mastogloia smithii THWAITES

Pleurosigma obscurum W. SMITH

Gyrosigma attenuatum (KUTZ) RABH

Gyrosigma spencerii (W. SMITH) CLEVE

Diploneis smithii (DE BREBISSON) CLEVE

Neiditum binode (EHR) HUSTEDT

Neidium $s p$.

Stauroneis $s p$

Stauroneis acuta W. SMITH

Anomoeoneis sphaerophora (EHR) PFITZER

Navicula cuspidata KUTZ

Navicula viridula KUTZ

Navicula rhynchocephala KUTZ

Navicula radiosa KUTZ

Navicula gracilis EHR

Navicula cryptocephala KUTZ

Navicula dicephala (EHR) W. SMITH

Navicula gastrum EHR

Navicula pupula KUTZ

Navicula seminulum GRUNOW

Caloneis amphisbaena (BORY) CLEVE

Pinnularia braunii (GRUN) CLEVE 
Pinnularia maior (KUTZ) CLEVE

Pinnularia viridis (NITZSCHE) EHR

Cymbella cuspidata KUTZ

Cymbella heteropleura EHR.

Cymbella aspera (EHR) CLEVE

Cymbella cistula (HEMPRICH) GRUN

Cymbella affinis KUTZ

Amphora ovalis KUTZ

Gomphonema acuminatum EHR

Gomphonema constrictum EHR

Gomphonema angustatum (KUTZ) RABH

Denticula sp.

Epithemia sorex KUTZ

Hankzschia amphioxys (EHR) GRUN

Bacillaria paxillifer (MULLER) HENDEY

Nitzschia scalaris (EHR) GRUN

Nitzschia sinuata W. SMITH

Nitzschia tryblionella HANTZSCH

Nitzschia hungarica GRUN

Nitzschia linearis W. SMITH

Nitzschia recta HANTZSCH

Nitzschia dissipata (KUTZ) GRUN

Nitzschia acuta HANTZSCH

Nitzschia gracilis HANTZSCH

Nitzschia pusilla KUTZ

Nitzschia palea (KUTZ) W. SMITH

Nitzschia romana GRUN

Nitzschia microcephala GRUN

Nitzschia amphibia GRUN

Nitzschia subcapitellata HUSTEDT

Nitzschia acicularis W. SMITH

Nitzschia closterium (EHR) W. SMITH

Nitzschia sigma (KUTZ) W. SMITH

Nitzschia filiformis (W. SMITH) HUSTEDT

Nitzschia acuminata (W. SMITH) GRUN

Nitzschia bilobata W. SMITH

Nitzschia hybrida GRUN

Nitzschia sigmoidea (EHR) W. SMITH

Cymatopleura solea (DE BREBISSON) W. SMITH

Cymatopleura elliptica (DE BREBISSON)

W. SMITH

Surirella ovata KUTZ

Campylodiscus sp.

\section{DINOPHYCEES}

Gymnodinium sp.

Peridinium $s p$.

Gonyaulax sp.

\section{EUGLENOPHYCEES}

Euglena sp.

Euglena viridis $\mathrm{EHR}$
Euglena acus (DUJ) HUTNER

Phacus sp.

\section{CYANOPHYCEES}

Aphanothece sp.

Chroococcus sp.

Anabaena sp.

Oscillatoria $s p$.

Pseudarabaena sp.

Lyngbya sp.

Microcoleus $s p$.

Pour chaque station, nous entendons par "peuplement global, l'ensemble des prélèvements réalisés tous les 12 jours pendant tout le temps de l'étude.

Dans les trois premières stations, qui sont les plus polluées, les Chlorophycées sont dominées par Chlamydomonas sp. qui forme $89 \%$ du peuplement global de cette classe en $\$ 1,91 \%$ en S2 et $45 \%$ en S3.

Les Cyanophycées sont essentiellement représentées par Oscillatoria sp. dont l'abondance constitue $88 \%$ de l'effectif de la classe en \$1, $86 \%$ en S2 et $90 \%$ en S3.

Dans la première station, parmi les Diatomées, Nitzschia palea domine $(74 \%$ du peuplement diatomique).

En S2, son abondance décroît (35\%) et Gomphonema angustatum (34\%). Navicula cryptocephala (23\%) réalisent ici une abondance relative élevée.

En S3, les abondances relatives sont plus faibles : Nitzschia palea (21\%), Navicula cryptocephala $(13 \%)$, Cocconeis placentula (13\%).

Dans les stations de l'aval, les Chlorophycées sont représentées par: Mougeatia sp. en $\$ 4$ ( $50 \%$ de l'effectif du groupe) et en S3A (72\%), Stigeoclonium sp. (18\%) et Oedogonium sp. (45\%) en S5, Ulothrix sp. en S6 (28\%), Oedogonium sp. en S7 (38\%).

Dans ces stations on note la présence de nombreuses espèces de Diatomées parmi lesquelles Nitzschia palea, Melosira varians, Navicula crypiocephala, Cocconeis placentula, Synedra ulna, Gomphonema angustatum sont généralement les plus abondantes.

\section{3 - Abondance relative moyenne}

Ce paramètre est obtenu de la façon suivante : on calcule, pour chaque prélèvement. l'abondance relative des différents groupes d'algues; on peut alors établir pour un même groupe taxonomique son abondance relative moyenne dans chaque station (ARM). 
Exemple :

AR M Diatumés $=\frac{A 1+A 2+\ldots . .+A N}{N}$

$\mathbf{N}=$ nombre total de prélèvements réalisés dans la station pendant les 5 mois.

A1, ..., $\mathbf{A N}=$ abondance relative des Diatomées dans les différents prélèvements ( 1 a $\mathrm{N})$ de la sta. tion considérée.

Pour chaque station, nous obtenons le spectre écologique de son peuplement (fig.4).

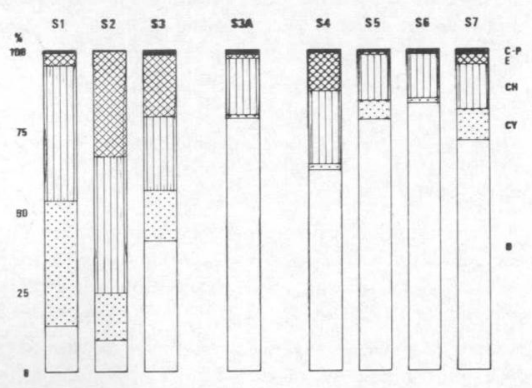

Fig 4. Variations stationnelles des abondances relatives moyennes, exprimées en \% de cellules, des différents groupes d'algues $(\mathbf{D}=$ Diatomées, $\mathbf{C Y}=$ Cyanophycées, $\mathbf{C H}=$ Chlorophycées, $\mathrm{E}=$ Euglénophycées, $\mathrm{C}$. $\mathbf{P}=$ Chrysophycées et Pyrrophycées).

Dans les trois premières stations, plusieurs classes sont bien représentées au cours de l'étude :

- en $\mathbf{S} 1$ dominent deux groupes, les Chlorophycées (42\%) et les Cyanophycees (39\%).

- en $\mathbf{S} 2$ les Chlorophycées présentent une abondance relative élevée $(43 \%)$ et sont numériquement suivies par les Euglénophycées ( $33 \%$ ).

- en S3 l'abondance relative des Diatomophycées augmente (41\%), celle des Chlorophycées décroît $(23 \%)$

Dans les stations de l'aval, dans le marais et en S3A, une seule classe, celle des Diatomophycées, domine (63 a $83 \%$ ).

Ainsi, dans les milieux pollués où les teneurs en $\mathrm{PO}_{4}-\mathrm{P}, \mathrm{NH}_{4}-\mathrm{N}$, MOD sont maximum et l'oxygénation réduite, on note une forte proportion de Chlorophy. cées, de Cyanophycées et d'Euglénophycées.
En aval, alors que se produit une amélioration progressive de la qualité de l'eau, ces trois classes cessent d'être dominantes et les Diatomées occupent alors la place prépondérante.

Dans les milieux aquatiques, de nombreux facteurs physico-chimiques influencent le développement et la composition des communautés algales (luminosité, température, vitesse du courant, chimisme des eaux) ; ceci a été exposé dans divers travaux (Blum 1956, Mc Intire 1968, Reisen \& al 1970. Patrick 1971).

D'une façon plus précise, certains auteurs ont montré que la distribution des différentes classes d'algues est directement liée à la composition chimique des eaux.

Ainsi, on signale dans les milieux enrichis en matière organique la présence de Chlamvdomonas sp. et d'Euglena sp. (Cairns et al 1972, Round 1973, Angely in Pesson 1976), de Cvanophycées (Fjerdings. tad 1965). Certains auteurs (Goldman et al. 1972. Round 1973) précisent que Chlamydomonas sp. et Euglena sp. peuvent devenir hétérotrophes facultatives. En revanche, les Diatomées sont généralement rencontrées dans des milieux moins pollués et plus oxygénés (Sladecek 1973, Angely in Pesson 1976).

La répartition des algues, dans le secteur étudié, confirme bien que la qualité physico-chimique des eaux détermine, en grande partie, la composition des communautés algales.

On observe ainsi le passage d'un peuplement hétérotrophe facultatif en zone polluée à un peuplement autotrophe composé essentiellement de Diatomées en aval de la pollution.

Le premier peuplement définit une zone polysaprobe (Zelinka \& Marvan 1961, Sladecek 1973). Le second peuplement comporte de nombreuses espèces de Diatomées (Nitzschia palea. Navicula cryptocephala, Melosira varians, Synedra ulna) que ces auteurs considèrent comme caractéristiques des zones mésoprobes.

\section{4 - Richesse spécifique moyenne}

C'est la movenne du nombre d'especes recensées dans chaque prélèvement, pour chaque station (RSM).

Exemple : R S M $=\frac{\mathrm{S}_{1}+\mathrm{S} 2 \ldots \ldots+\mathrm{SN}}{\mathrm{N}}$

$\mathrm{N}=$ nombre total de prélèvements réalisés dans la station pendant les 5 mois. 
$\mathbf{S} 1, \ldots, \mathbf{S N}=$ nombre d'espèces des différents prélèvements ( 1 à $\mathrm{N}$ ) de la station considérée.

La richesse spécifique moyenne, faible dans les deux premières stations, augmente progressivement en aval et atteint son maximum en S5. En S6 et en S7 les valeurs diminuent. La station $\mathrm{S} 3 \mathrm{~A}$, non perturbée, est caractérisée par un nombre d'especes peu élevé, proche de celui rencontré en $\$ 7$ (fig. 5).

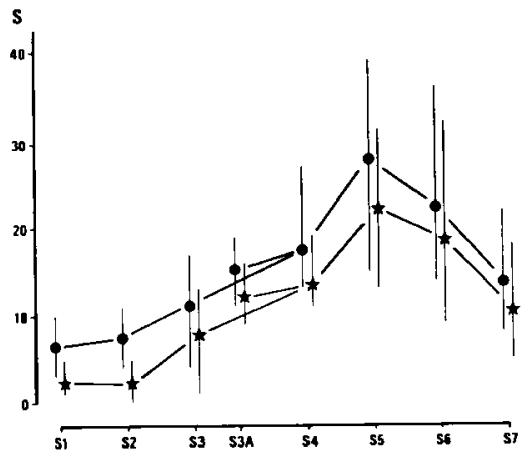

Fig 5. Richesses spécifiques (S) : moyennes et amplitudes de variations (ensemble du peuplement algal • _ * peuplement diatomique $\star$ _ $\star$ ).

La richesse moyenne en Diatomées suit une évolution parallèle; faible dans les trois premières stations, mais augmentant de $\mathrm{S} 2$ à $\mathrm{S} 3$, elle atteint une valeur maximum dans le marais (S5), (fig. 5).

\section{5 - Indice de diversité et équitabilité}

L'équation de Shannon \& Weaver (Daget 1976) a été appliquée pour définir la structure du peuplement algal. Nous avons déterminé, dans chaque station, la moyenne des indices de diversité et des équitabilités calculés pour chaque prélèvement (fig. 6).

Les indices de diversité et les équitabilités les plus faibles correspondent aux secteurs les plus pertubés S1, S2 et soulignent l'instabilité de la structure du peuplement algal à ce niveau. En aval, les valeurs des indices de diversité et d'équitabilité augmentent. Elles reflètent une distribution plus uniforme des individus entre les taxons et une structure du peuplement donc plus équilibrée.

Les valeurs des indices dans les stations de l'aval témoignent ainsi d'une meilleure stabilité du milieu

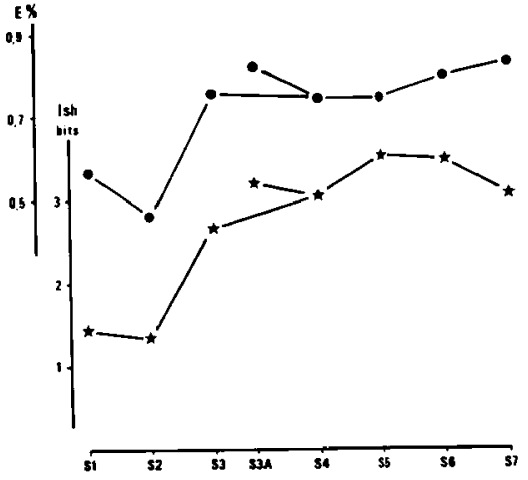

FIG 6. Indices moyens de diversité et d'equitabilité : Ish $\star$ Ł $\mathrm{E} \bullet-$

et traduisent l'amélioration de la qualité physicochimique et biologique de l'eau.

L'évolution spatiale de l'indice de diversité est identique à celle observée par Cooper \& Wilhm (1975) en milieu pollué et en secteur peu perturbé.

\section{5. - Discussion et conclusion}

Dans les deux premières stations, S1 et S2, les teneurs élevées en matières organiques dissoutes, en éléments nutritifs et les faibles taux d'oxygène, éliminent de nombreuses espèces. Seules celles qui sont tolérantes à la pollution organique se développent. Ce sont essentiellement des algues hétérotrophes facultatives qui colonisent les substrats. La variété spécifique étant faible, la compétition entre espèces est réduite : ainsi, chaque population atteint une forte densité.

En aval, l'amélioration des conditions de milieu élimine progressivement les Euglénophycées, les Volvocales et les Cyanophycées ; par contre, la communauté des Diatomées devient plus riche en espèces. Les concentrations en éléments nutritifs étant réduites et la richesse spécifique élevée, une sévère compétition s'établit ent re les espèces limitant ainsi le développement de chacune (Patrick 1950, Patrick \& Strawbridge 1963).

Dans la station S3A, non polluée, où les Diatomées sont peu abondantes et peu diversifiées, on peut sup- 
poser que les faibles teneurs en éléments nutritifs limitent le développement de ces algues.

Au niveau de la station $\mathrm{S} 7$, située sous un couvert végétal dense et dans laquelle les teneurs en éléments dissous sont voisines de celles mesurées en S6, les taux de chlorophylle "a", la densité du peuplement diatomique et la diversité spécifique y sont généralement plus faibles. Dans cette station où l'énergie incidente est réduite, la luminosité interviendrait en tant que facteur limitant de la densité et de la diversité du peuplement algal.

Dans les stations S3 et S4, situées dans une zone de transition réunissant les caractères chimiques des deux milieux (S1-S2 d'une part, le marais d'autre part), la réduction du peuplement algal à ce niveau indique que les conditions hydrochimiques sont peu favorables à la croissance, tant des algues autotrophes que des algues hétérotrophes.

Ainsi, dans le secteur étudié, on observe le pas. sage d'un peuplement algal à dominantes hétérotrophes facultatives (Euglénophycees, Volvocales) de faible diversité en milieu très pollué, à un système autotrophe diversifié et composé essentiellement de Diatomées en zone moins perturbée.

La succession des communautés algales et les variations de la structure des peuplements stationnels reflètent donc l'évolution de la charge polluante ; celle-ci, par ailleurs, dépend en grande partie du métabolisme de ces mémes algues (utilisation des sels nourriciers en excès) qui ainsi participent activement aux processus d'épuration des eaux.

Au terme de cette étude, on constate que les com. munautés algales en intégrant les variations de la qualité des eaux renseignent sur les perturbations subies par le milieu et deviennent donc un critère biologique précieux dans l'étude des pollutions des cours d'eau.

Enfin, sur le plan méthodologique cette étude souligne, une fois de plus, l'intérêt de l'utilisation des substrats artificiels qui permettent d'obtenir d'une façon commode, peu onéreuse, une image du peuplement algal très satisfaisante dans sa composition et sa structure.

\section{Remerciements}

Nous tenons à exprimer notre vive gratitude à Monsieur le Professeur P. Bourrelly (Museum, Paris) qui a bien voulu vérifier nos déterminations, chaque fois que nous l'avons sollicité.

\section{Travaux cltés}

Blum (J.L.). 1956. - The ecology of river algae. But, Rev., 22 (5) $291 \cdot 341$.

Cairns (J.J.), Lanza (G.R.) \& Parker (B.C.). 1972.- Pollution related structural and functional changes in aquatic communities with emphasis on freshwater algae and protozoa. Proceed. Acad. Nat. Sci. Phi., 124 (5) : 79 - 127.

Capblancq (J.) \& Cassan (M.). 1979.- Etude du périphyton d'une rivière polluce (I'Agout). I. Structure et développement des communautés sur substrats artificiels. Annls Limnol, 15 (2) $193 \cdot 210$.

Cooper (J.M.) \& Wilhm(J.). 1975.-Spatial and temporal variation in productivity, species diversity, and pigment diversity of periphyton in a stream receiving domestic and oil refinery effluents. Southwest. Nat., 19 (4) : 413 - 428 .

Daget (J.). 1976. - Les modèles mathématiques en écologie. Masson, Paris: $172 \mathrm{p}$.

Fjerdingstad (E.). 1965. - Taxonomy and saprobic valency of benthic phytomicro-organisms. Int. Revue ges hydrobiol., 50 (4) $475 \cdot 604$.

Giudicelli (J.), Dia (A.) et Légier (P.) 1980.- Etude hydrobiologique d'une rivière de région méditerranéenne, I'Argens (Var, France). Habitats, hydrochimie, distribution de la faune ben. thique. Bijdragen tot de Dierkunde, 50 (2) : 303 . 341.

Goldman (J.C.), Porcella (D.B.), Middlebrooks (E.J.) \& Toerien (D.F.) 1972. - The effect of carbon on algal growth. Its relationship to eutrophication. Water research, $6: 637+679$.

Lorenzen (C.J.). 1967. - Determination of chlorophyll and pheopig ments : Spectrophotometric equations. Limnol. Oceanogr., 12 (2) : $343 \cdot 346$

Martinez-Ansemil (E.). 1978. - Structure horizontale des communautés d'invertèbrés benthiques d'une rivière méditéraneenne, l'Argens (Var). Influence du substrat et du courant. These 3e cycle, Aix-Marseille III, $145 \mathrm{p}$.

Mc Intire (C.D.). 1968. - Structural characteristics of benthic algal communities in laboratory streams. Ecology, 49 (3) : 520 - 537.

Millerioux (G.). 1974 - 1975. - Comparaison de méthodes spectrophotométriques pour le calcul des pigments phytoplanctoniques. Annls Stat. Biol Besse-en-Chandesse, 9:59-77.

Patrick (R.). 1950.- Biological measure of stream conditions. Sew. Ind. Wastes, 22 (7) $926-938$.

Patrick (R.) \& St rawbridge (D.). 1963.- Variation in the structure of natural diatom communities. Amer. Nat., 97 (892): 51 - 57.

Patrick (R.). 1971. - The effects of increasing light and temperature on the structure of diatom communities. Limnol. Ocea nogr., $16(2): 405-421$

Pesson (P.). 1976.- La pollution des eaux continentales. Incidence sur les biocénoses aquatiques. Gauthier-Villars, Paris. 285 p.

Reisen (W.K.) \& Spencer (D.J.). 1970.- Succession and current demand relationships of diatoms on artificial substrates in Prater's Creek, South Carolina. J. Phycol. 6:117 - 121.

Round (F.E.). 1973. - The Biology of the Algae. Arnold London, 20 édition. $278 \mathrm{p}$

Sladecek (V.). 1973. - System of water quality from the biological point of view. Ergebnisse der Limnologie., 7: 218 p.

Zelinka (M.) \& Marvan (P.). 1961.- Zur Präzisierung der biologischen Klassifikation der Reinheit fliessender Gewässer. Arch. Hydrobiol, 57 (3) : 389 - 407. 